\title{
In Vivo Activation by Ultraviolet Rays of the Human Immunodeficiency Virus Type 1 Long Terminal Repeat
}

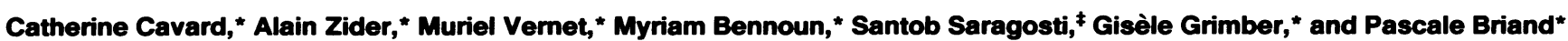
* Génétique et Pathologie Expérimentales; ${ }^{\ddagger}$ Immunologie et Oncologie des Maladies Retrovirales, Unité 152 Institut Cochin de Génétique Moléculaire, Institut National de la Santé et de la Recherche Medical, F-75014, Paris, France

\begin{abstract}
It has been previously shown in vitro that the human immunodeficiency virus type 1 long terminal repeat (LTR) is activated by ultraviolet irradiation. In order to analyze if a similar effect could occur in vivo, transgenic mice carrying the $\operatorname{lac} Z$ gene under the control of the viral LTR were irradiated at 280-300 and $254 \mathrm{~nm}$. These mice spontaneously expressed the transgene in the epidermis and the lens of both adults and embryos. Irradiations caused a significant increase in skin $\beta$-galactosidase activity. This phenomenon might be involved in viral activation and could be of interest in regard to the skin pathology observed during an HIV infection. (J. Clin. Invest. 1990. 86:1369-1374.) Key words: transegenic mice • HIV-1 • UV • keratinocytes
\end{abstract}

\section{Introduction}

A variety of clinical syndromes, including AIDS, neurological disorders, and Kaposi's sarcoma may follow as a consequence of infection with the human immunodeficiency virus type 1 (HIV-1). ${ }^{1}$ This etiological agent was shown to infect CD4 ${ }^{+} \mathrm{T}$ lymphocytes, cells of the macrophage-monocyte lineage and of the central nervous system (1). One of the characteristics of this prototype human lentivirus is the extended time frame, for months to years, from initial HIV infection to clinically detectable immunologic abnormalities and disease manifestations. Previous studies have shown that in addition to the HIV-1 regulatory proteins, physical, chemical, and other viral agents can act in vitro to activate the HIV-1 long terminal repeat (LTR) (2-4). Since activation signals are required for the establishment of the productive HIV infection in vitro, it is likely that activators contribute to the conversion of a latent or chronic infection to a productive one in vivo. The identification of factors that induce in vivo viral expression could be of use in controlling the evolution of the disease.

Address reprint requests to Dr. Catherine Cavard, Génétique et Pathologie Expérimentales, ICGM 22, rue Méchain, F-75014, Paris, France.

Received for publication 16 March 1990.

1. Abbreviations used in this paper: CAT, chloramphenicol acetyltransferase; HIV-1, human immunodeficiency virus type 1 ; LTR, long terminal repeat; UV, ultraviolet.

J. Clin. Invest.

(c) The American Society for Clinical Investigation, Inc.

$0021-9738 / 90 / 10 / 1369 / 06 \$ 2.00$

Volume 86, October 1990, 1369-1374
Transgenic mice carrying the viral LTR controlling the expression of a reporter gene can be used for studying the cellular and exogenous factors that may activate in vivo the HIV-1 LTR. We have generated transgenic mice carrying an insert in which the U3R region of the HIV-1 LTR is linked to the bacterial gene $l a c Z$ which encodes for the $\beta$-galactosidase protein. This reporter gene allows a rapid and precise localization of expression.

We present in this report the spontaneous activity of the viral LTR in various tissues of transgenic mouse models and the evidence of its activation in the skin by ultraviolet (UV) B and $C$ rays.

\section{Methods}

Construction of the chimeric gene. A 722-bp Hind III restriction fragment containing the U3R region $(-642-+80)$ (BRU isolate) excised from the plasmid pLC2S was inserted at the unique Hind III site of plasmid pCH110 (Pharmacia Fine Chemicals, Uppsala, Sweden) upstream of the $\beta$-galactosidase sequence. The insert $(5,230 \mathrm{bp})$ containing the U3R region, the $l a c Z$ gene, and the SV 40 polyadenylation signal was excised from bacterial plasmid sequences by digestion by Nco I and Pst I.

Production of transgenic mice. Before microinjection into fertilized eggs, the insert was purified from the vector sequences on a low melting point agarose gel and isolated by dissolution in $0.6 \mathrm{M}$ sodium iodide on 325 mesh silica. The fragment was diluted for injection to $3.5 \mathrm{ng} / \mu \mathrm{l}$ in $10 \mathrm{mM}$ Tris- $\mathrm{HCl}$ pH 7.5, 0.1 mM EDTA. DNA ( 300-500 copies per egg) was microinjected using established procedures into fertilized eggs resulting from mating between B6D2F1 hybrids (5).

DNA analysis. 2-wk-old animals were screened for the insertions of the U3R-lacZ hybrid gene by Southern blot analysis of tail DNA preparations. Genomic DNA's $(10 \mu \mathrm{g})$ were digested with Hind III and separated by electrophoresis through $1 \%$ agarose gels, then transferred to Zetabind filters (AMF-Cuno, Meriden, CT). Prehybridization and hybridization were carried out according to the supplier's recommendations. The probe was the microinjected fragment (Fig. $1 B$ ) labeled by the random priming method (6). Copy number was determined by dot blot analysis.

Escherichia coli $\beta$-galactosidase assay. Whole specimens or skin biopsies were flash frozen in isopentane in liquid nitrogen and mounted in OCT compound (Miles Laboratories Inc., Naperville, IL) for cryosectioning. Sections $(10 \mu \mathrm{m})$ were immediately fixed $10 \mathrm{~min}$ in 4\% p-formaldehyde in 0.1 M PBS pH 7.2. After rinsing in PBS, slides were incubated overnight at $30^{\circ} \mathrm{C}$ in a buffer containing $2 \mathrm{mM} \mathrm{X}$-gal (5-bromo-4-chloro-3-indolyl- $\beta$-D-galactoside), $4 \mathrm{mM}$ potassium ferricyanide, $4 \mathrm{mM}$ potassium ferrocyanide, $4 \mathrm{mM}$ magnesium chloride in 0.1 M PBS. Sections were stained with hematoxylin and eosin according to standard methods.

$U V$ irradiation of mice. Areas $\left(1.5 \mathrm{~cm}^{2}\right)$ of tail from control and transgenic mice were irradiated with a UV B lamp (Philips fluores- 


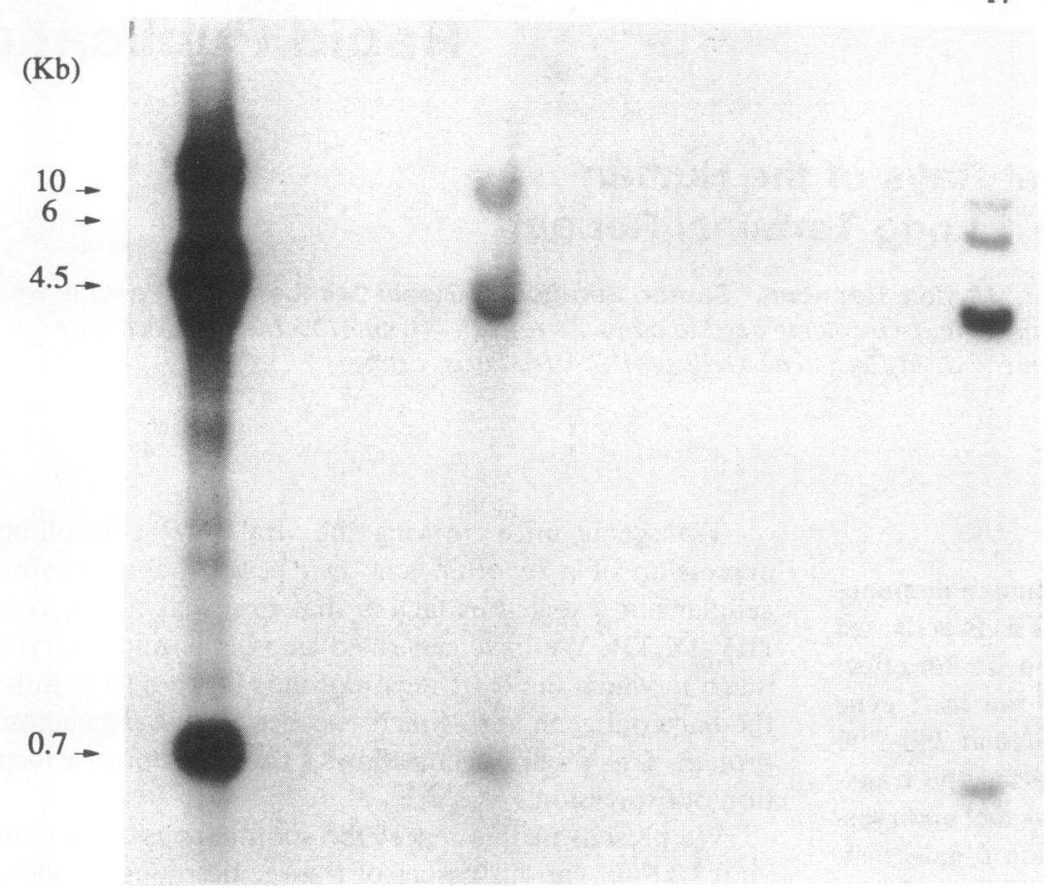

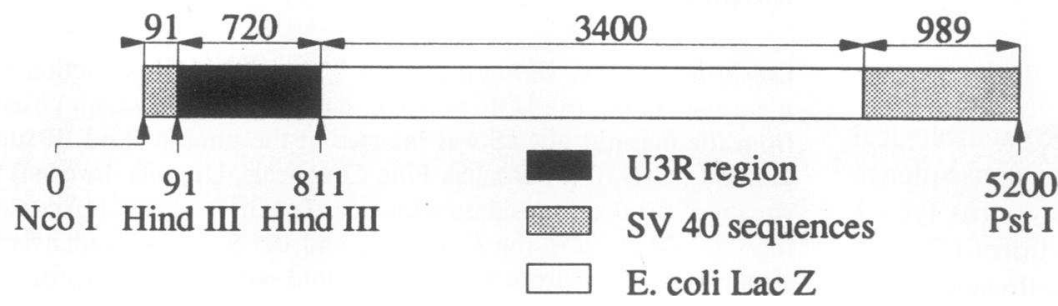

Figure 1. $(A)$ Detection of transgenic mice by Southern blot analysis. DNA samples of 17 newborn mice were digested with Hind III. The microinjected fragment described in $(B)$ was used as a probe. The sizes of DNA fragments are indicated on the left. $(B)$ Restriction map of the microinjected fragment. cence tube TL 20, 280-320 nm, 0.1 J/m²; Philips Electronic Instruments, Mahwah, NJ) or an UV C lamp (Flufrance, $254 \mathrm{~nm}, 2 \mathrm{~J} / \mathrm{m}^{2}$ ). Tail biopsies were performed before irradiation and $24,48 \mathrm{~h}$, and $6 \mathrm{~d}$ after.

\section{Results}

Generation of $U 3 R$-lacZ transgenic mice. Southern blot analysis of restriction enzyme-digested DNA from the tails of 18 live-born animals revealed that three contained integrated copies of the hybrid gene (Fig. $1 A$ ). In transgenic lines 6, 10, and 17 , the transgene was organized as a head to tail tandem array. Copy number ranged from $\sim 5$ to 100 (data not shown). Analysis of the progeny of these founder animals showed that the transgene was transmitted at a frequency of $\sim 50 \%$.

Expression of the transgene in the adult mice. Expression of the hybrid gene was assayed by a sensitive enzymic in situ detection procedure for $E$. coli $\beta$-galactosidase, in which the chromogenic substrate, $\mathrm{X}$-gal, is enzymically cleaved by $\beta$-galactosidase to release an insoluble blue dye. Expression was first investigated in the adult mice by examination of cryostat sections for $\beta$-galactosidase activity. Among all the organs tested (brain, thymus, heart, lung, liver, spleen, pancreas, kidney, muscles, skin, and eye), the skin and the eyes spontaneously expressed the transgene. The same pattern of expression was detected in two independent strains.
In the eye, the $\beta$-galactosidase activity was restricted to some fiber lens cells (Fig. $2 a$ and $b$ ) located in the cortical region of the lens known to contain the most recently formed fiber cells during embryonic development. In the skin, expression was limited to the epidermis and was associated with both hair follicles and cells interspersed throughout the stratum corneum (Fig. $2 c$ and $d$ ). In both cases, the majority of the cells producing the protein were anucleated, suggesting that they were at the ultimate stage of differentiation. Expression in the skin was variable according to the sites of biopsies. The activity was reduced in fur-bearing skin (dorsal and ventral side of the mice) compared with the ears and the tail.

Expression of the transgene in the embryos. The same pattern of expression observed in adults was found in the embryos of the two strains expressing the transgene. Activity in the eyes was detectable from gestational age (G) 11 and persisted until birth. At G 15, the staining in the lens was clearly visible even without sectioning as it is shown in Fig. $3 a$. Examination of cryostat sections showed that $\beta$-galactosidase activity was located in a few lens fibers (Fig. $3 b$ and $b^{\prime}$ ). Besides the lens staining, an intense external staining of the embryo was apparent. Examination of fetuses revealed that the lacz gene was strongly expressed over almost the entire skin surface. In addition to the keratinized peridermal cells, a few cells surrounding the hair follicle were also stained (Fig. $3 c$ ). The $\beta$-galactosidase 

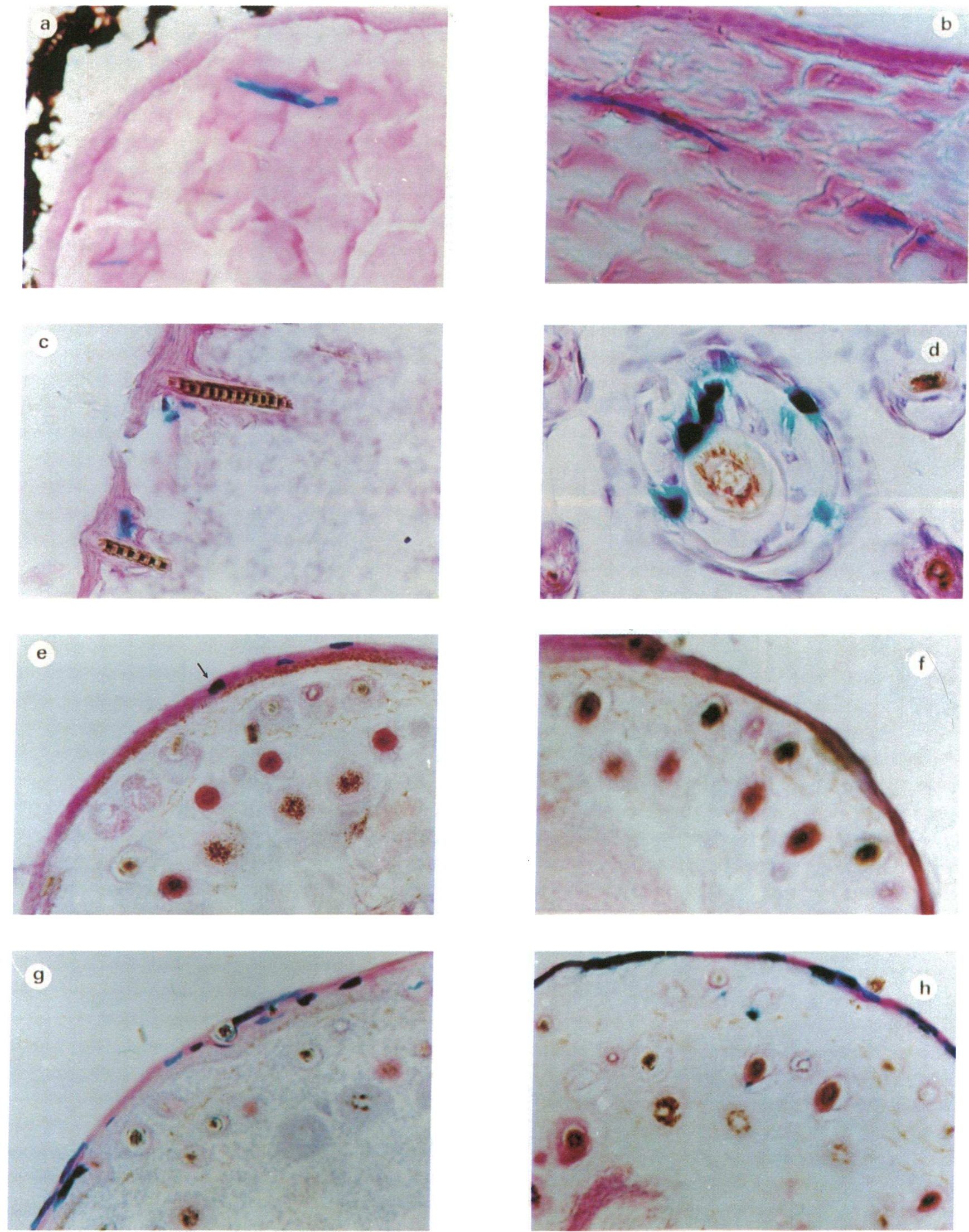

Figure 2. In situ assay of bacterial $\beta$-galactosidase activity in sections prepared from transgenic mouse line. The line 17 was chosen for its highest $\beta$-galactosidase activity. (a) Transverse section of an eye of 6-mo-old transgenic mouse; $25 \times .(b)$ idem $\times 62.5$. (c) Transverse section of dorsal skin of a 1-mo-old transgenic mouse; $62.5 \times .(d)$ Cross section of dorsal skin of a 1-mo-old transgenic mouse; $125 \times$. (e- $h)$ Transverse sections of tail of 10-d-old mice; $25 \times$. (e) Transgenic control mouse. $(f)$ Negative control mouse. $(g)$ Transgenic mouse UV C irradiated $(60 \mathrm{~J}) 48$ $\mathrm{h}$ after exposure. $(h)$ Transgenic mouse UV B irradiated $(60 \mathrm{~J}) 24 \mathrm{~h}$ after exposure. 

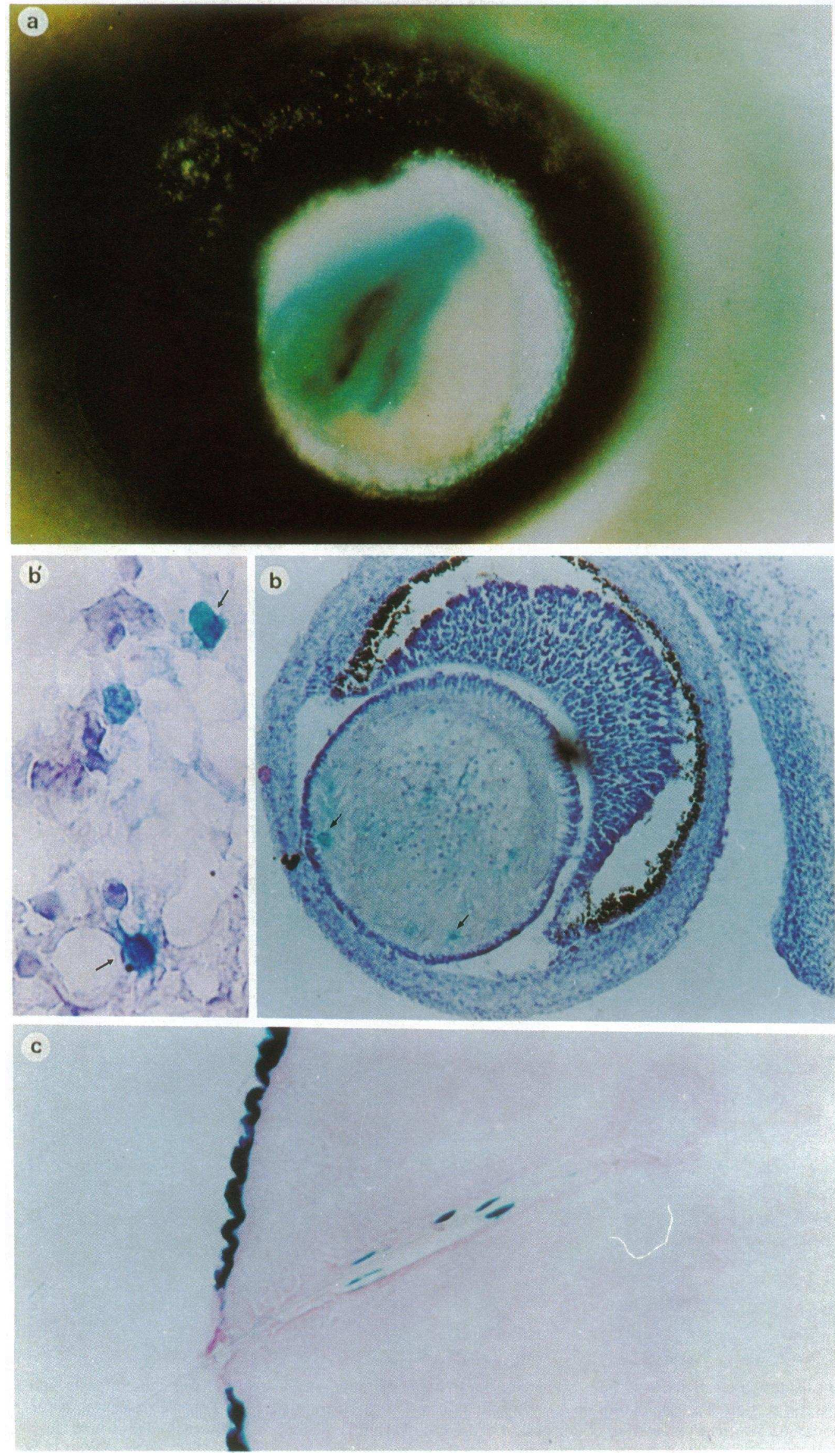

Figure 3. In situ assay of bacterial $\beta$-galactosidase activity in $\mathrm{G} 15$ embryos of the transgenic line 17. (a) Whole eye; $4 \times$. (b) Sagittal section of the eye; $25 \times$. $\left(b^{\prime}\right)$ Sagitta section of the eye; $187 \times .(c)$ Transverse section of skin; $25 \times$. 
activity in peridermal cells was constant all through the gestation and staining was markedly more elevated than that observed in epidermal keratinocytes after birth.

$U V$-induced gene expression. Previous in vitro experiments led to the hypothesis that UV irradiation could affect HIV-1 expression in vivo $(4,7,8)$. To test this hypothesis, irradiations at 280-320 (UV B rays) and $254 \mathrm{~nm}$ (UV C rays) were performed on 10-d-old mice. In both cases, a significant increase in $\beta$-galactosidase activity compared with control animals was observed on skin biopsy sections (Fig. $2 g$ and $h$ ). This effect was detected within $24 \mathrm{~h}$ after irradiation and persisted for 24 $h$ at the same level. The activating effect was obtained in a dose-dependent manner with a peak at $60 \mathrm{~J}$ after $10 \mathrm{~min}$ or 30 s of exposure under UV B and UV C irradiation, respectively. Expression was shown to decrease at increasing doses up to 180 (i.e., $30 \mathrm{~min}$ ) and $90 \mathrm{~J}$ (i.e., $45 \mathrm{~s}$ ) for UV B and UV C, respectively. $6 \mathrm{~d}$ later, the activating effect had completely disappeared even though an epidermic hyperplasia associated with a marked hyperkeratosis was still detectable. No effect had been detected with UV A irradiation.

\section{Discussion}

We generated three transgenic lines carrying the HIV-1 LTRlac $Z$ transgene. In one strain $(n=6)$, the absence of any detectable activity suggested that the injected sequence had incorporated into a region of inactive chromatin or sustained an undetectable sequence alteration. The pattern of expression was characterized in the two other independent strains by a marked $\beta$-galactosidase activity in the lens and in the skin and thus appeared to be conferred by the HIV-1 LTR. The pattern of expression in these transgenic mice carrying a U3R-lacZ transgene is similar to the one described in another transgenic model in which the HIV LTR is linked to the chloramphenicol acetyltransferase (CAT) gene. In that model, expression was also observed in the thymus (9). We cannot exclude the possibilities that a low level of $\beta$-galactosidase production in this organ would not be detected by the staining method used or that this enzyme would be less stable in this tissue than the CAT protein. The level of mRNA encoded by the transgene in the thymus is now under investigation by in situ hybridization. The transgene expression found in the skin and the eyes clearly confirmed that an expression driven by the HIV-1 LTR could be possible in vivo even in the absense of the viral transactivator TAT protein. This suggests that in vivo specific cellular factors could supply the action of TAT protein.

The direct in situ detection of the enzymatic activity in our model has allowed us to locate precisely in which cells of the epidermis the HIV-1 LTR directed expression could occur. The expressing cells were predominantly keratinocytes by nature of their terminal differentiation. A high activity was also observed around the hair follicles. Altogether, these localizations might explain some of the pathological modifications (epidermal hyperplasia and particularly nodular proliferation of mature keratinocytes in submandibular masses) observed in transgenic mice carrying the entire HIV-1 genome (10). Furthermore, transgenic mice carrying the LTR-TAT transgene exhibit, as the earliest lesion in the skin, an epidermal hyperplasia before the development of a Kaposi's like syndrome (11).

In the previously reported LTR-CAT model (9), the CAT activity was detected predominantly in the Langerhans cells but also in keratinocytes. If we assumed that the reporter genes, CAT and lacZ, were neutral and the pattern of expression was specific of the HIV-1 LTR, an explanation for this difference could be that the in vitro culture step used by Leonard et al. (9) might induce CAT expression in Langerhans cells. We might suggest that expression in these cells needs in vitro serum factors found in the culture medium and, in vivo, viral factors secreted by the HIV-infected cells. In vitro experiments are currently being performed on our system to test this hypothesis. Furthermore, the method generally used for preparation of epidermal cells might not allow a satisfying recovery of a representative population of cells of the epidermis. Our preliminary results indicated that trypsination used for the separation of the epidermis led preferentially to the dissociation of basal and suprabasal cells. The cornified cells which expressed the transgene remained associated with the epidermal sheath.

Under UV induction, the cell types in which the staining was detected were in the same location as those in which the HIV-LTR was spontaneously active, that is, in the external cornified layer of the epidermis. UV irradiation might act directly on keratinocytes or indirectly by promoting the secretion of an extracellular factor by irradiated cells, which in turn may activate the HIV-1 transcription in other cell types, as suggested by in vitro experiments (7-8). Like a number of eucaryotic promoters such as $c$-fos, metallothionein, and collagenase, known to be susceptible to DNA damaging agents and phorbol esters (7), retroviral promoters might be activated during the cascade of events triggered by the stress response. It has been recently reported that such activations by $U V, x$ ray, and phorbol myristate acetate could occur in vitro for the Moloney murine sarcoma virus LTR (12). This effect is mediated by activation of the protein kinase $C$ and would be independent of newly synthesized proteins. In vivo activation reported in this work appeared to follow the same kinetics of response to UV stimulation as has been described in vitro for both viruses, although their final mechanisms of activation could be different.

Pathological phenomena induced by UV rays in HIV-infected cells could be enhanced by a cumulative effect linked to the DNA damages produced, and to the downregulation of DNA reparation processes presumably induced by some lentivirus. Host chromosomal damage has been recently correlated with viral gene expression in experiments showing that the transactivator gene of the HTLV-1 virus downregulated in vitro the expression of human $\beta$-polymerase involved in host cell DNA repair (13).

Our results demonstrate that UV B (and UV C) rays can activate HIV-1 expression in vivo. The doses described in our model are severalfold higher than those previously described for cultured cells, but may be a better representation of the natural phenomenon which occurs during a prolonged exposure to sunlight. These results also suggest that UV B irradiation might be involved in viral activation, triggering an expression of HIV-1 sequences in cell types different from those previously shown to be the major source of the virus in the skin, and explaining some of the skin pathology observed during HIV-1 infection.

\section{Acknowledgments}

We thank M. Rizk and W. T. Stringfellow for advice and discussion.

This work was supported by grants from ANRS and ARC (No. 


\section{References}

1. Fauci, A. S. 1988. The human immunodeficiency virus: infectivity and mechanisms of pathogenesis. Science (Wash. DC). 239:617622.

2. Twu, J. S., K. Chu, and W. S. Robinson. 1989. Hepatitis B virus $X$ gene activates K-B like enhancer sequences in the long terminal repeat of human immunodeficiency virus 1. Proc. Natl. Acad. Sci. USA. 86:5168-5172.

3. Ensoli, B., P. Lusso, F. Schachter, S. F. Josephs, J. Rappaport, F. Negro, R. C. Gallo, and F. Wong Staal. 1989. Human herpes virus-6 increases HIV-1 expression in co-infected $\mathrm{T}$ cells via nuclear factors binding to the HIV-1 enhancer. EMBO (Eur. Mol. Biol. Organ.) J. 8:3019-3027.

4. Kristoffer, V., A. Delers, C. Bruck, C. Thiriart, H. Rosenberg, C. Debouck, and M. Rosenberg. 1988. Activation of human immunodeficiency virus type 1 by DNA damage in human cells. Nature (Lond.). 333:78-81.

5. Gordon, J. W., and F. H. Ruddle. 1983. Gene transfer into mouse embryos: production of transgenic mice by pronuclear microinjection. Methods Enzymol. 101:411-433.

6. Feinberg, A. P., and B. Vogelstein. 1983. A technique for radiolabeling DNA restriction endonuclease fragments to high specific activity. Anal. Biochem. 132:6-13.

7. Stein, B., H. J. Rahmsdorf, A. Steffen, M. Litfin, and P. Herrlich. 1989. UV-induced DNA damage is an intermediate step in UV-in- duced expression of human immunodeficiency virus type 1, collagenase, c-fos, and metallothionein. Mol. Cell. Biol. 9:5169-5181.

8. Stein, B., M. Krämer, H. J. Rahmsdorf, H. Ponta, and P. Herrlich. 1989. UV-induced transcription from the human immunodeficiency virus type 1 (HIV-1) long terminal repeat and UV-induced secretion of an extracellular factor that induces HIV-1 transcription in nonirradiated cells. $J$. Virol. 63:4540-4544.

9. Leonard, J., J. S. Khillan, H. E. Gendelman, A. Adachi, S. Lorenzo, H. Westphal, M. A. Martin, and M. S. Meltzer. 1989. The human immunodeficiency virus long terminal repeat is preferentially expressed in Langerhans cells in transgenic mice. AIDS (Lond.). 5:421-430.

10. Leonard, J. M., J. W. Abramczuck, D. S. Pezen, R. Rutledge, J. A. Belcher, F. Hakim, G. Shearer, L. Lamperth, W. Travis, T. Fredrickson, A. L. Notkins, L., and M. M. Martin. 1988. Development of disease and virus recovery in transgenic mice containing HIV proviral DNA. Science (Wash. DC). 242:1665-1670.

11. Vogel, J., S. H. Hinrichs, R. K. Reynolds, P. A. Luciw, and G. Jay. 1988. The HIV tat gene induces dermal lesions resembling $\mathrm{Ka}-$ posi's sarcoma in transgenic mice. Nature (Lond.). 335:606-610.

12. Lin, C. S., D. A. Goldthwait, and D. Samols. 1990. Induction of transcription from the long terminal repeat of Moloney murine sarcoma provirus by UV-irradiation, $x$-irradiation, and phorbol ester. Proc. Natl. Acad. Sci. USA. 87:36-40.

13. Jeang, K. T., S. G. Widen, O. J. Semmes, and S. H. Wilson, 1990. HTLV-1 trans-activator protein, tax, is a transrepressor of the human $\beta$-polymerase gene. Science (Wash. DC). 247:1082-1084. 